A. Manchado ${ }^{1}$, S.R. Pottasch ${ }^{2}$, and A. Mampaso ${ }^{1}$

1. Instituto de Astrofisica de Canarlas, Tenerife, Spain

2. Kapteyn Astron. Institute, Groningen, The Netherlands

ABSTRACT. Long slit low resolution (3.4 A) spectra of the planetary nebulae NGC 6543 and NGC 6826, obtained using the 2.5-m Isaac Newton Telescope (La Palma) with the Image Photon Counting System (IPCS), indicate different physical conditions in the outer halos than in the central zone, with an outward increase of electronic temperature. The estimated mass contained in these halos is considerably larger than the values of the inner nebulae.

The calculated chemical abundances seem lower in the halos than in the central parts.

From the above considerations it is clear that the emission of the halos of these nebulae is thermal and not reflection by dust.

\title{
EXPANSION VELOCITIES OF SOUTHERN PLANETARY NEBULAE
}

K.C. Sahu and S.R. Pottasch

Kapteyn Laboratorium, Groningen, The Netherlands

ABSTRACT. We have undertaken a programme of kinematic study of southern planetary nebulae by obtaining high-resolution ( $\mathrm{x} \geqq 50,000)$ spectra, using the ESO 1.5-m telescope + the Coude Echelle Spectrograph. As first results of this study, this paper presents previously unknown expansion velocities of 16 planetary nebulae. This result increases the total number of planetary nebulae for which the expansion velocities are now known by about $10 \%$. Further, reliable distance measurements and other physical properties are available for most of these sources. Hence this sample significantly improves the previously available data for statistical analysis related to the dynamics and evolution of planetary nebulae. Some preliminary results based on such statistical analysis are presented. The details of the results will be published in Astron. Astrophys. 